\title{
UNE FILAIRE NOUVELLE DU HÉRON CENDRÉ \\ (ARDEA CINEREA L.) LEMDANA URBAINI N. SP.
}

\author{
Par Y. CAMPANA
}

L'autopsie d'un héron cendré de la Ménagerie du Muséum d'Histoire naturelle de Paris (1) nous a permis de récolter des filaires immatures sur les aponévroses de la cavité générale. Ces filaires paraissent bien appartenir au genre Lemdana Seurat 1917, mais nous n'avons pu les rattacher à aucune espèce connue. Nous en donnons ici la description.

Matériel : quatre femelles et deux mâles.

L'hôte a dû s'infester en captivité, sinon il faudrait admettre 'que le développement de ces filaires demande plus d'un an.

Description. - Nématode blanc opalescent, s'amincissant aux deux extrémités. Cuticule mince, à stries transversales fines et serrées $(1,3 \mu$ environ). Champs latéraux larges de $20 \mu$, nettement visibles. Pas d'ailes latérales.

Extrémité antérieure atténuée régulièrement; orifice buccal allongé dorso-ventralement, dépourvu de lèvres, limité par un mince bourrelet. Quatre papilles submédianes peu importantes au cycle externe ; deux amphides plates situées latéralement et un peu en arrière. Cette disposition rappelle tout à fait celle de $L$. lomonti Desportes 1947. Diérides non appréciables; nous avons cru les discerner sur un seul exemplaire, à $42 \mu$ de l'apex.

Esophage nettement divisé en deux parties. Dans la portion glandulaire élargie, la lumière de l'œsophage se trouve déplacée du côté ventral.

\section{Femelle}

Nous donnons les dimensions d'une femelle de $5,7 \mathrm{~mm}$., les futres ayant respectivement $5,3 \mathrm{~mm}$., $5,5 \mathrm{~mm}$. et $5,8 \mathrm{~mm}$.

Largeur : $320 \mu$.

(1) Nous remercions bien vivement M. le Professeur Achille Urbain qui a bien voulu nous donner ce héron pour une expérience sur le cycle évolutif d'Avioserpens galliardi.

Ann. de Parasitologie, $\mathrm{T}$ XXIV, $\mathrm{N}^{0 \mathrm{~s}}$ 5-6. - 1949, p. 443-446. 
Protorhabdion : $30 \mu$.

Esophage musculaire : $230 \mu$.

Esophage glandulaire : $450 \mu$.

L'intestin, mince sur toute sa longueur, se termine par un rectum court.

Anus fonctionnel, à $70 \mu$ de l'extrémité postérieure.

Anneau nerveux et pore excréteur bien visibles, respectivement à 160 et $265 \mu$ de l'apex.

Vulve à $680 \mu$ de l'extrémité antérieure, surmontée d'un petit mamelon faisant saillie sur la face ventrale; ovéjecteur long de $550 \mu$; utérus de $325 \mu$ de long sur $125 \mu$ de large, se divisant postérieurement en deux branches parallèles dirigées vers l'arrière, mais dont l'une se replie à $2,15 \mathrm{~mm}$. de l'apex, pour aboutir à l'ovaire antérieur qui forme une boucle en avant de l'utérus, à $1 \mathrm{~mm}$. de l'extrémité antérieure, et se termine à $1,9 \mathrm{~mm}$. de celle-ci. L'ovaire postérieur forme une boucle à $550 \mu$ de l'extrémité postérieure et se termine à $1,9 \mathrm{~mm}$. de celle-ci.

Ces dimensions peuvent varier considérablement chez une femelle mature : ici, l'appareil génital ne contient pas d'œufs; seule la femelle de $5,8 \mathrm{~mm}$. présente des masses cellulaires encore très peu différenciées. Nous n'avons pu mettre en évidence la présence de papilles caudales.

\section{Mâle}

Longueur : $4,35 \mathrm{~mm}$. et $3,7 \mathrm{~mm}$. Les dimensions ci-dessous sont celles du plus grand exemplaire :

Protorhabdion : $28 \mu$.

Esophage musculaire : $200 \mu$.

Esophage glandulaire : $430 \mu$.

Pore excréteur : $200 \mu$.

Le testicule se replie à $650 \mu$ de l'extrémité antérieure.

Spicules très inégaux : le spicule gauche, long et mince, peu chitinisé, mesure $450 \mu$, le spicule droit, court et trapu, $125 \mu$.

Nous n'avons pu voir les papilles cloacales décrites pour certaines espèces.

Discussion. - Les principaux caractères de notre filaire sont les suivants :

$1^{\circ}$ extrémité antérieure nue, sans épaulettes ;

$2^{\circ}$ œsophage en deux portions bien distinctes ;

$3^{\circ}$ queue très courte ;

$4^{\circ}$ spicules très inégaux ; 
$5^{\circ}$ parasite du tissu conjonctif de Ciconiiforme.

Parmi les Filarioidea connus à l'heure actuelle, seul le genre Lemdana Seurat 1917 nous paraît correspondre à cette description.
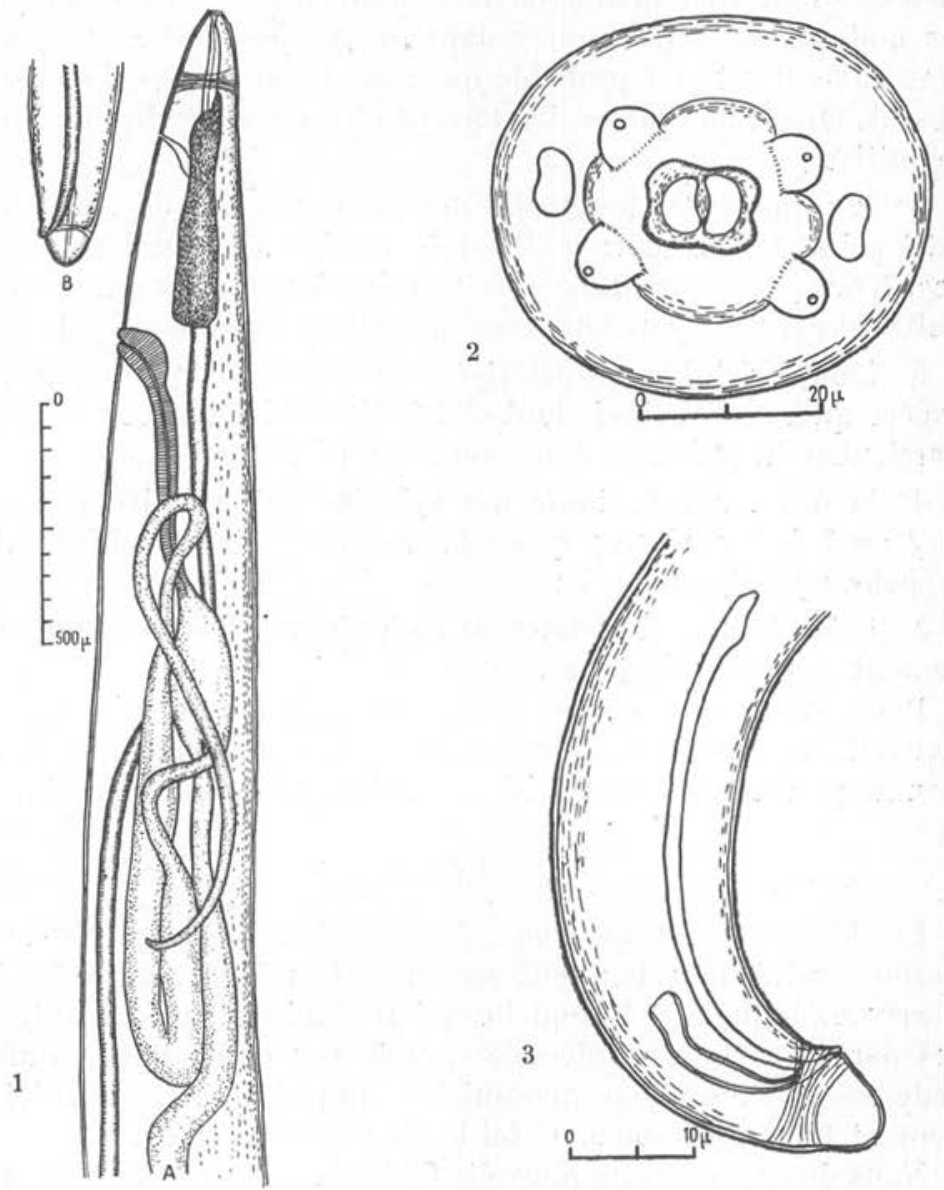

Fıc. 1. - Lemdana urbaini n. sp., femelle. A, extrémité antérieure ; B, extrémité postérieure.

FIG. 2. - Lemdana urbaini n. sp., femelle. Tête, vue apicale.

F1G. 3. - Lemdana urbaini n. sp., mâle, exłrémité postérieure.

Nos exemplaires étant immatures, il est assez difficile de les comparer avec ceux des espèces connues; cependant, un certain nombre de caractères nous permettent de les différencier :

$1^{\circ}$ les quatre espèces bien décrites que nous connaissons sont beaucoup plus grandes que la nôtre : les femelles mesurent $32 \mathrm{~mm}$. 
chez L. limboonkengi Hœppli et Hsü 1929, $34 \mathrm{~mm}$. chez L. marthæ Seurat 1917, $37 \mathrm{~mm}$. chez L. lomonti Desportes 1947, et 69 à $94,5 \mathrm{~mm}$. chez $L$. behningi Lewaschoff 1930 . Les mâles mesurent 10 à $12,8 \mathrm{~mm}$. chez L. marthæ et $26 \mathrm{~mm}$. chez L. behningi. Les femelles matures peuvent grandir dans des proportions assez considérables, mais il est peu probable qu'elles atteignent les dimensions cidessus. Quant aux mâles, ils doivent être assez proches de leur taille définitive ;

$2^{\circ}$ le rapport des longueurs des deux portions de l'œsophage est ici à peine de $1 / 2$, alors qu'il est de $1 / 6$ à $1 / 12$ pour les autres espèces. L'œsophage glandulaire se termine à peu près au niveau de la vulve, il est bien plus long chez les autres espèces ;

$3^{\circ}$ l'amphidelphie caractérisée ne permet de comparer notre espèce qu'à $L$. behningi, dont elle diffère par d'autres caractères, en particulier la présence d'un bourrelet péribuccal ;

$4^{\circ}$ la différence de taille des spicules est relativement faible: $\mathrm{d} / \mathrm{g}=1 / 3,5$ environ ; pour L. marthæ : $\mathrm{d} / \mathrm{g}=1 / 11$, et pour L. behningi : $\mathrm{d} / \mathrm{g}=1 / 7$;

$5^{\circ}$ enfin, le pore excréteur est bien visible et assez grand, contrairement aux autres espèces.

Pour toutes ces raisons, nous croyons pouvoir créer une espèce nouvelle, à laquelle nous donnons le nom de Lemdana urbaini, en hommage reconnaissant à M. le Professeur Achille Urbain.

\section{RÉSUMÉ}

Les filaires que nous avons récoltées dans la cavité générale d'un héron cendré appartiennent au genre Lemdana Seurat 1917. Nous décrivons le mâle et la femelle qui se différencient des autres espèces par un certain nombre de caractères : petite taille, dimensions réduites de l'œesophage glandulaire, amphidelphie, inégalité relativement faible des spicules, taille du pore excréteur.

Nous donnons à cette nouvelle filaire le nom de Lemdana urbaini.

\section{BIBLIOGRAPHIE}

Desportes (C.). - Tridel phie chez une filaire nouvelle parasite c̉u héron pourpré (Ardea purpurea L.). Ann. Parasit., XXII, 1947, 36-44.

HoEppli (R.) et Hsu (H. F.). - Parasitische Nematoden aus Vögeln und einem Tïmmler. Arch. Schiffs- u. Tropenhyg., Beihefte, XXXIII, 1929, 24-34.

Lewaschoff (M.). - Beitrag zur Kenntnis der Fauna der Parasitischen Nematoden des unteren Wolgagebietes. Zeitschr. Parasitenk., II, 1930, 121-128.

Sevrat (L.-G.). - Nématodes de la perdrix de roche. Bull. Soc. Hist. Nat. Afr. Nord, VIII, 1917, 208-215. 